3. Kapikian AZ, James HD Jr, Kelly SJ, et al. Isolation from man of "avian infectious bronchitis virus-like" viruses (coronaviruses) similar to 229E virus, with some epidemiological observations. J Infect Dis 1969;119:282-290.

4. Calderaro A, Arcangeletti MC, De Conto F, et al. SARS-CoV-2 infection diagnosed only by cell culture isolation before the local outbreak in an Italian seven-week-old suckling baby. Int J Infect Dis 2020;96:387-389.
5. Zhou J, Li C, Liu X, et al. Infection of bat and human intestinal organoids by SARS-CoV-2. Nat Med 2020;26:1077-1083.

6. La Scola B, Le Bideau M, Andreani J, et al. Viral RNA load as determined by cell culture as a management tool for discharge of SARS-CoV-2 patients from infectious disease wards. Eur J Clin Microbiol Infect Dis 2020;39:1059-1061.

\title{
Unrecognized severe acute respiratory coronavirus virus 2 (SARS-CoV-2) seroprevalence among healthcare personnel in a low-prevalence area
}

\author{
Vishal P. Shah MD ${ }^{1}$, Caitlin M. Hainy APRN, CNP, DNP², Melanie D. Swift MD, MPH ${ }^{1,2}$, Laura E. Breeher MD, MPH ${ }^{1,2}$, \\ Elitza S. Theel $\mathrm{PhD}^{3}$ and Priya Sampathkumar MD ${ }^{4}$ \\ ${ }^{1}$ Division of Preventive, Occupational, and Aerospace Medicine, Mayo Clinic, Rochester, Minnesota, ${ }^{2}$ Occupational Health Services, Mayo Clinic, Rochester, \\ Minnesota, ${ }^{3}$ Department of Laboratory Medicine and Pathology, Division of Clinical Microbiology, Mayo Clinic, Rochester, Minnesota and ${ }^{4}$ Division of Infectious \\ Diseases, Mayo Clinic, Rochester, Minnesota
}

To the Editor-Healthcare personnel (HCP) caring for patients with severe acute respiratory syndrome coronavirus 2 (SARS-CoV-2) may have higher rates of coronavirus disease 2019 (COVID-19) than other HCP. A study of asymptomatic HCP in Texas found much higher rates of SARS-CoV-2 reverse-transcriptase PCR (RT-PCR) positivity in COVID-19-facing HCP (4.8\%) compared to other HCP $(0.6 \%){ }^{1}$ However, RT-PCR evaluation alone may lead to an underestimation of COVID-19 infections due to testing only symptomatic cases, timing of sample collection, and/or false-negative tests. ${ }^{2}$ Antibody assessment could provide further insight into the prevalence of COVID-19 among HCP. Immunoglobulin G (IgG) antibodies to SARS-CoV-2 typically develop within 8-14 days of symptom onset, and most are seropositive by 21 days. ${ }^{3}$ A multistate study of frontline HCP revealed significant heterogeneity in seroprevalence, ranging from $0.8 \%$ to $31.2 \%$; however, HCP who tested positive by RT-PCR were also included. ${ }^{4}$ Our study sought to determine the seroprevalence of HCP who did not previously test positive for COVID-19 by RT-PCR at a tertiary-care medical center in the midwestern United States.

Mayo Clinic employees in Rochester, Minnesota, were recruited between May 25 and July 9, 2020, and were placed into high- and low-risk cohorts based on their role and work unit. The high-risk cohort included HCP in a direct patient care role, working in the emergency department, COVID-19 intensive care unit, or COVID-19 general care ward. Low-risk HCP were those not involved in direct patient care HCP who previously tested positive or who had a household member previously test positive for COVID-19 were excluded from the study. All study participants

\footnotetext{
Author for correspondence: Vishal P. Shah, E-mail: Shah.vishal1@mayo.edu

Cite this article: Shah VP, et al. (2021). Unrecognized severe acute respiratory coronavirus virus 2 (SARS-CoV-2) seroprevalence among healthcare personnel in a low-prevalence area. Infection Control \& Hospital Epidemiology, 42: 1532-1534, https:// doi.org/10.1017/ice.2020.1341
}

completed a questionnaire about occupational and community exposure and were asked about symptoms consistent with COVID-19. This study was approved by the Mayo Clinic Institutional Review Board (no. 20-003787).

All enrolled HCP were first screened using the Euroimmun anti-SARS-CoV-2 IgG ELISA (Lubeck, Germany). ${ }^{5}$ Positive or indeterminate samples were retested using either the Roche Diagnostics anti-SARS-CoV-2 Total Antibody Immunoassay (Roche Diag nostics, Rotkreuz, Switzerland) or the Ortho-Clinical Diagnostics anti-SARS-CoV-2 IgG Antibody Immunoassay (Ortho-Clinical Diagnostics, Raritan, NJ). Only participants with positive tests by 2 assays were considered seropositive for antibodies to SARS-CoV-2. RT-PCR testing records were assessed through occupational health records from March 9 through June 30, 2020. The $\chi^{2}$ and Fisher exact tests were used for the statistical analysis.

In total, 586 participants were enrolled, and 568 completed the survey and laboratory analysis (Table 1). Overall, 2 of $320(0.63 \%)$ $\mathrm{HCP}$ in the high-risk cohort tested positive for IgG antibodies against SARS-CoV-2, while 0 of $248(0 \%)$ tested positive in the low-risk cohort $(\mathrm{OR}, 3.90 ; P=.51)$. The 2 individuals with antibodies to SARS-CoV-2 did not report breaches in personal protective equipment (PPE), nor were they advised to quarantine due to a known exposure to a person with COVID-19. One seropositive study participant reported symptoms of chills, myalgias, diarrhea, and a headache, and the second participant reported a headache. A higher number of HCP in the high-risk cohort were placed on a quarantine due to known exposure to a person with COVID-19 compared to the low-risk cohort (odds ratio [OR], 12.1; $P=.0016$ ).

Based on role and employment location, there were 1,348 employees who would have been eligible to enroll in the high-risk cohort. From March 9 to June 30, 2020, 7 of these employees tested positive by RT-PCR, 3 of whom had known community exposures. The RT-PCR positive rate between the high-risk cohort $(0.52 \%)$ and the non-high-risk cohort $(0.57 \%)$ were similar and were also comparable to overall RT-PCR prevalence rates in the region $(0.67 \%$ as of July 1,2020$){ }^{6}$

(c) The Author(s), 2020. Published by Cambridge University Press on behalf of The Society for Healthcare Epidemiology of America. This is an Open Access article, distributed under the terms of the Creative Commons Attribution licence (http://creativecommons.org/licenses/by/4.0/), which permits unrestricted re-use, distribution, and reproduction in any medium, provided the original work is properly cited. 
Table 1. Demographic Information and Survey Analysis

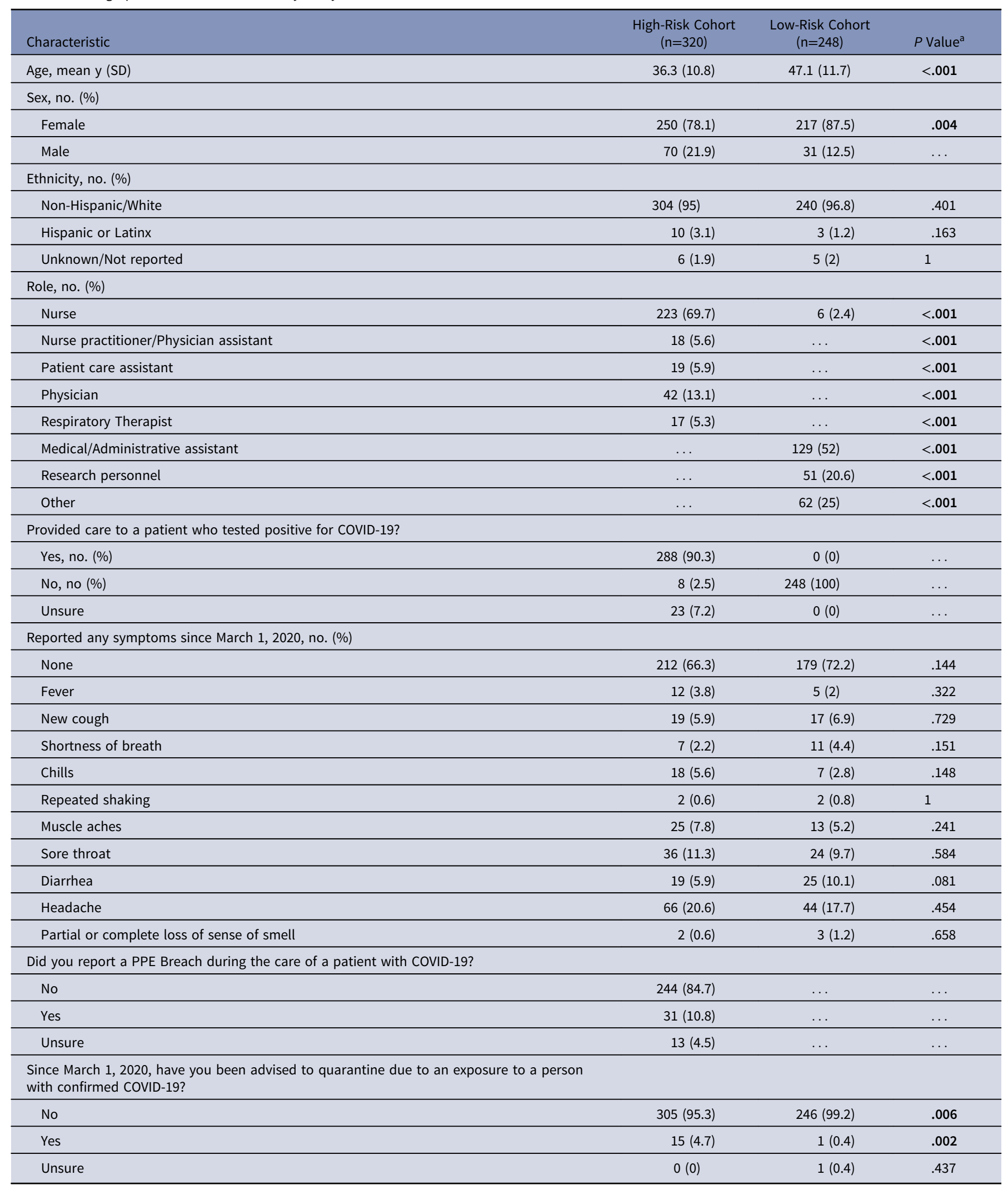

${ }^{a}$ Student $t$ test was used for continuous variables and the Fisher exact test was used for categorical variables. 
Previous data describing COVID-19 infection rates in HCP have been mixed. The multistate study of frontline HCP and other studies have shown significant heterogeneity among frontline HCP that generally correlated with community rates. ${ }^{4} \mathrm{~A}$ study evaluating IgG antibodies to SARS-CoV-2 among HCP in Germany found the overall rate of unrecognized prior infection to be $1.6 \%{ }^{7}$ However, the use of single antibody assays in low-prevalence areas may lead to high rates of false-positive results. A Centers for Disease Control and Prevention (CDC) study assessing antibody prevalence across 10 sites in the United States from March 23 to May 12, 2020, revealed highly variable prevalence rates ranging from $1 \%$ to $6.9 \% .^{8}$ An orthogonal testing algorithm was utilized; however, the study did not specify whether individuals had been symptomatic or previously tested by RT-PCR.

Here, we present serology and RT-PCR data to determine the prevalence of recognized and unrecognized COVID-19 infections among HCP. In this low-prevalence setting, HCP in the high-risk cohort are much more likely to encounter persons with COVID-19 in the occupational setting compared to the community. Despite providing care to patients with COVID-19 and having higher odds of being quarantined due to an exposure to a person with COVID19 , the rate of SARS-CoV-2 infection based on combined seroconversion and RT-PCR positivity did not differ between high- and low-risk HCP, and they were similar to community rates.

Our study has several limitations. Given the kinetics of antibody development against SARS-CoV-2, individuals tested shortly after infection may not have mounted an antibody response. Additionally, the county in which the hospital is situated also had a low prevalence of COVID-19, as determined by the percentage of positive by RT-PCR tests.

Overall, HCP regularly caring for patients with COVID-19 did not have significantly higher rates of COVID-19 infection compared to other HCP at a tertiary-care center in Minnesota. In particular, rates of unrecognized infection were low. These data support the efficacy of current processes to identify and isolate
COVID-19 patients and to limit HCP exposure to COVID-19 through administrative practices, training, and rigorous use of PPE.

Acknowledgments. We would like to thank our study coordinater, Katherine B. Erickson, for her contribution and support of this work.

Financial support. No financial support was provided relevant to this article.

Conflicts of interest. E.S.T. reports serving on the Advisory Board of Roche Diagnostics and Accelerate Diagnostics and receiving research funding from Ortho-Clinical Diagnostics. All other authors report no conflicts of interest relevant to this article.

\section{References}

1. Vahidy FS, Bernard DW, Boom ML, et al. Prevalence of SARS-CoV-2 infection among asymptomatic healthcare workers in the greater Houston, Texas, area. JAMA Netw Open 2020;3(7):e2016451.

2. He X, Lau EHY, Wu P, et al. Temporal dynamics in viral shedding and transmissibility of COVID-19. Nat Med 2020;26:672-675.

3. Fang FC, Naccache SN, Greninger AL. The laboratory diagnosis of coronavirus disease 2019-frequently asked questions. Clin Infect Dis 2020. doi: 10. 1093/cid/ciaa742.

4. Self WH, Tenforde MW, Stubblefield WB, et al. Seroprevalence of SARSCoV-2 among frontline healthcare personnel in a multistate hospital network-13 academic medical centers, April-June 2020. Morb Mortal Wkly Rep 2020;69:1221-1226.

5. Theel ES, Harring J, Hilgart H, Granger D. Performance characteristics of four high-throughput immunoassays for detection of IgG antibodies against SARS-CoV-2. J Clin Microbiol 2020;58(8). doi: 10.1128/JCM.00512-20.

6. COVID-19 Weekly Report. Olmsted County, Minnesota, website. https:// www.co.olmsted.mn.us/OCPHS/COVID-19/Pages/default.aspx. Published 2020. Accessed November 20, 2020.

7. Korth J, Wilde B, Dolff S, et al. SARS-CoV-2-specific antibody detection in healthcare workers in Germany with direct contact to COVID-19 patients. J Clin Virol 2020;128:104437-104437.

8. Havers FP, Reed C, Lim T, et al. Seroprevalence of antibodies to SARS-CoV-2 in 10 sites in the United States, March 23-May 12, 2020. JAMA Intern Med 2020. doi: $10.1001 /$ jamainternmed.2020.4130. 